\title{
Class Characteristic Classification of Test Fired Cartridge Cases: A Digital Image Decision Tree Approach to Kennington's Matrix for Initial Stages of Criminal Investigation
}

\author{
Young Wang* \\ Department of Forensic \& Investigative Science, West Virginia University, USA
}

Submission: November 23, 2017; Published: November 30, 2017

*Corresponding author: Young Wang, Department of Forensic \& Investigative Science, West Virginia University, USA; Email: yowang@mix.wvu.edu

Abstract

For a no-gun scene, an investigator faces a challenge in determining what gun to submit to the lab when he doesn't know what type of gun was possibly involved. However, there exists no detailed electronic class characteristic database for spent cartridge cases in either the current GRC or NIBIN system. Using purposive sampling, the author fired eight different popular models of $9 \mathrm{~mm}$ semiautomatic firearms in an indoor shooting range and collected 130 fired cartridge cases/casings. A digital portable microscope was used and a digital image classification system was created based upon Kennington's matrix. The preliminary results indicate value in four practical ways:

a) The image classification database is convenient and user friendly to create;

b) The classification database can be varied in size, ranging from an examiner's personal use to a lab-to-lab based system;

c) The database allows for a quick reference search for an investigative purpose;

d) The database can serve as a quick reference for previously examined test fires for purposes of court testimony. It is suggested if the image classification database is to be adopted, determinations of the type of gun based upon the type of cartridge case would be much more convenient, easy, and practical for criminal investigations.

Keywords: Firearms and Tool marks examination; Kennington class characteristic matrix; No-gun crime scene; 9mm Casings; Type/model determination; Bivariate statistics; Variance-covariance

Abbreviations: GRC: General Rifling Characteristic; AFTE: Association of Firearms and Tool mark Examiner; IBIS®: Integrated Ballistics Identification System ${ }^{\circledR}$

\section{Introduction}

When a gun is fired, ammunition components are transferred to a crime scene. The ammunition components alone are a source of information about the gun fired. One piece of information about the gun is the broad class of caliber, such as a $9 \mathrm{~mm}$, for semi-automatic pistols. But what is more important in a no-gun crime scene for criminal investigations "What was the make/ model of $9 \mathrm{~mm}$ gun used?" While ammunition components such as bullets are currently in an electronic General Rifling Characteristic (GRC) database maintained by the FBI for this exact purpose of possible gun type determination, the list of brands generated is often quite lengthy.
There currently exists no equivalent detailed electronic database for spent cartridge cases. Thus, a database and non-cumbersome standardized procedure for determining the brand of gun which fired a cartridge case based on detailed class characteristics is much needed in order for investigators and examiners to have images and quantitative measurements to support conclusions such as "what make/model is most likely." An attention to keeping up to date with more detailed class characteristics which were specific to a particular model and make of firearm as it entered the market was clearly evident in the early 1990s. Nordhoff solely dedicated to a new model of .25 ACP Lorcin L25 Pistols [1]. The company Lorcin itself had just been established 
less than a year prior and was marketing inexpensive firearms targeted towards lower income individuals.

The designs of the firearms were similar to corresponding models made by another earlier manufacturer Raven Arms. Specifically, both the L25 and the MP-25 were both 7-shot pocket pistols designed to be easily concealed. Nordhoff published the similarities and important differences in the class characteristics of these similar firearms which would likely become "Saturday night special" firearms distributed through pawn shops and eventually be used in the commission of a crime [1]. In response to Nordhoff, Thompson published a similar article in 1991 on class characteristics, but for the $10 \mathrm{~mm}$ Wyoming Arms Parker [2]. One reason for a $10 \mathrm{~mm}$ handgun to make its way in the commission of a crime can be seen given the historic move of the FBI towards .40 Smith \& Wesson (S\&W) in 1990. The .40 S\&W was the replacement for the higher recoil $10 \mathrm{~mm}$ cartridge and delivered the desired terminal ballistics in a cartridge which could be chambered in smaller frame handguns.

Later in 1991, Gieszl argued the need for examiners to update their class characteristic files for the new Model 83 and 85 handguns chambered in .380 ACP manufactured by Argentine arms maker Bersa [3]. Once again, the 83 and 85 were examples of affordable and concealable firearms which would be popular and potentially end up being used in crimes. The significance of selecting firearms based on market models and subsequently the likelihood in casework is to provide for an examiner an update his/her lab's personal reference files of the detailed class characteristics. As a result, the firearm could then be easily referred to in future case work involving no-gun cases. Finally in 1992, Carr re-examined the class characteristics of the Lorcin L25 for the unique way it marked the shoulder of bullets due to the lead or gap between the firearm's chamber and button rifled barrel [4]. Again, in a no-gun case, knowledge of these class characteristics would allow the investigator and the examiner to narrow down the make and model of firearm.

\section{The Kennington Matrix System}

Perhaps the greatest advancement for class characteristic prediction of firearms during this time was Robert Kennington's publication of his book "The Matrix; 9 mm Parabellum: An Empirical Study of Type Determination." Kennington and also his lab had been using physical cards for class characteristics since the 1950s. The cards were organized into a matrix by manufacturer and type of bullet, chamber marks, extractor mark shape, ejector mark, firing pin impression, and breech face marks. In some instances, Kennington was able to narrow the possibility of manufacturers down to one with the caveat there could exist a manufacturer which was not in the database [5].

Practically, the police detective who is armed with the hypothesized manufacturer at the time of interrogating a suspect is more likely to get a meaningful confession, particularly if the suspect believes his accomplice has already confessed. This confession could than lead to other evidence such as the actual firearm itself to be used for test firing as well as additional incriminating evidence such as the buried body which is needed to secure a guilty verdict if a case were to go to trial. However, the format of using cards to update the database has proved to be not only inconvenient but also outdated because new firearms entered the market almost annually. Therefore, there exists a need for an electronic version which still captures the essence of the original method but in a more quantitative format for easier searching and greater objectivity.

The last major effort of earlier type determination on class characteristics in the literature was perhaps a book review of Kennington's previously discussed works on $9 \mathrm{~mm}$ and .380 ACP done by Dutton, of the Tasmania Police in 2003 [6]. According to Dutton, Kennington's decade old work contained not only a great deal of technical information but was still relevant to the current time. Specifically, Dutton considered the potential workflow of how shooting crimes should be investigated and how labs encountering $9 \mathrm{~mm}$ and $.380 \mathrm{ACP}$ on a regular basis ought to devote time now for implementation of Kennington's method in order to save time later in the future [6]. It is such encouragement which serves and inspires the basis for this research project.

\section{Current Lab Databases and Systems}

One electronic class characteristic database for a fired bullet would be the General Rifling Characteristic (GRC) database maintained by the FBI. The GRC database is for the number of and numerical widths of lands and grooves which were engraved on a bullet when the bullet engaged the rifling of the barrel it was fired through. However, a detailed class characteristics database for cartridge cases in an electronic format to facilitate easier information sharing amongst examiners is long overdue as GRC databases are often insufficient for no-gun cases. As Oberg observed for a $9 \mathrm{~mm}$ caliber right twisted bullet with seven lands and grooves and a cartridge case with hemispherical firing pin impression, two extractor marks, and one ejector mark: the initial search of the GRC database returned zero hits [7] Nevertheless, it was additional research which was found to be more useful using the online Association of Firearms and Tool mark Examiner (AFTE) forum which suggested the firearm may have been made by SCCY. Oberg then had to reach out to the manufacturer SCCY in order to deduce the type of firearm as being a model CPX-1 with class characteristic 4 o'clock extractor and 7-8 o'clock ejector [7].

Another electronic system which is commonly misunderstood by non-firearm examiners is the Integrated Ballistics Identification System ${ }^{\circledR}$ (IBIS $®$ ) system. Past public policy has also been slightly misguided with respect to using cartridge case databases powered by computer algorithms such as IBIS ${ }^{\circledR}$ in an attempt to preliminarily identify or individualize a particular gun as opposed to a broader class of make/model. While IBIS ${ }^{\circledR}$ is useful for linking different shootings to one another and to an already seized firearm (where a one-to- 
one comparison by a trained human examiner is still needed thereafter for identification conclusions for court). IBIS $₫$ will not deliver a list of manufacturers. The related state governments perhaps misunderstood its capabilities when they had respectively passed costly legislature to mandate every new gun legally sold to have test fired cartridge cases entered into an IBIS $\AA$ powered database. Thus, there currently exists no electronic database of fired cartridge cases for generating investigative leads in the common situation where a gun has yet to be recovered. Meanwhile, there are many gun manufacturers introducing new models of guns every year, making it difficult to update such a database unless a cost-effective, non-cumbersome standardized procedure is developed.

\section{Research Design}

\section{A Model Digital Image Database}

Purposive sampling was employed to select firearms, in this case, the $9 \mathrm{~mm}$ semiautomatic pistols, with three major considerations: first, the $9 \mathrm{~mm}$ is the most common or mode of firearms used in crimes nationwide; second, the availability of the firearm in local indoor shooting ranges; and lastly, the personal experience and expertise of the author. As a result, the following seven models of popular recoil action operated $9 \mathrm{~mm}$ models were selected
a) The Smith \& Wesson M\&P9,
b) Sig Sauer P226,
c) Springfield XD9,
d) Ruger SR9,
e) Beretta 92FS,
f) CZ 75, and
g) HK USP.

The seven models of $9 \mathrm{~mm}$ pistols were test fired in an indoor shooting range using the standard factory Winchester "white box" 115 grain with Full Metal Jacket ammunition. Glock firearms such as the 17 and 19 chambered in $9 \mathrm{~mm}$ which produced a rectangular firing pin aperture mark were not studied due to the widespread knowledge of this signature class characteristic.

Glocks such as the Glock 43 and the latest Generation 5 Glock which do not produce this signature rectangular firing pin aperture mark are an object of future study and comparison. Additionally, one additional blowback operated Ruger Police Carbine 9 was test fired for an anticipated study and comparison with the popular blowback operated Hi-Point C9 handgun and 995TS carbine in the near future. Ten test fired cartridge cases from each firearm were randomly selected and analyzed using a method-based to what was discussed by Warren and Pitts [8]. While Warren and Pitts placed each cartridge case image in a 4X4 grid in a Microsoft PowerPoint slide in order to quantify the location of an ejector mark in a meaningful manner, this method was considered to be too imprecise and does not address the original challenge referenced by Kennington in 1992 [5]. This challenge is the need for collaborative sharing. Collaborative sharing is not only important for practitioners since new firearms are constantly being manufactured, but also for the broader legal and academic communities, since continued confusion and criticism of the discipline is still prevalent and rooted in a lack of understanding and transparency over the existence of personal databases for known test fired cartridge cases.

Rather than using a $4 \times 4$ grid for the entire cartridge case, a $24 \times 24$ grid was used just focusing on the small pistol 4.45 $\mathrm{mm}$ primer with the 24 grid units corresponding to or covering $4.45 \mathrm{~mm}$ primer area. To put this in perspective, each one-half grid unit corresponded to $0.0927 \mathrm{~mm}$ or less than $100 \mathrm{um}$. The cropping of only the primer area was done because ejector marks are often not present and the center of mass of irregularly shaped ejector marks is more difficult to determine as compared to a firing pin impression which is always present and symmetric. However, the original uncropped images are still retained within the same folder and a slide show can still be used not only for one-to-one comparisons of ejector mark shapes as opposed to using an algorithm [9] which may not be able to handle a multiclass problem as well as the human eye, but also to assess the visual variability within an individual firearm as well as within a make/model.

Next, when firing pin drag marks were present, they were used to orient the cartridge case so the drag mark pointed up towards 12 o'clock or $(0,1)$ in an $x-y$ coordinate system. Similarly, when firing pin drag marks were absent, the extractor marks were used to orient the cartridge case so the extractor mark pointed towards 3 o'clock or $(1,0)$. The center of the grid is the origin $(0,0)$ of the primer. The center of the firing pin impression was determined by placing the mouse over the center of the firing pin impression, reducing the transparency of the image until the background grid lines were visible. The coordinate was then recorded as the number of grid units away from the center in the horizontal $\mathrm{x}$-axis direction (negative corresponding to left, positive corresponding to right) followed by the number of grid units away from the center in the vertical y-axis direction (negative corresponding to low, positive corresponding to high). Independent of rotation, the numerical distance between the center of the firing pin and the center of the primer was determined using the Pythagorean Theorem.

\section{Results}

\section{The Google Drive/Slides/Sheets Platform}

While Warren and Pitts chose to use Microsoft Power point, this was found to be less useful than using Google Slides since the ultimate goal is to facilitate sharing. Google Slides is Google's version of Power point and exists within an individual or organization's Google Drive, Google's cloud-based file storage and sharing platform. Although Microsoft does offer their version, One Drive, Google Drive was used since it is the default 
cloud storage system used by the author's institution of study: West Virginia University. Within Google Slides, a template for measurements was created by selecting a 4:3 aspect ratio for the slide and adding 33 evenly spaced lines in the horizontal direction and 25 evenly spaced lines in the vertical direction for the generation of 32 by 24 boxes ( $4: 3$ ratio), where only the center 24 by 24 boxes were used (to correspond to a circular primer area). The two lines composing the $\mathrm{x}$ and $\mathrm{y}$ axes were highlighted in red (Figure 1).

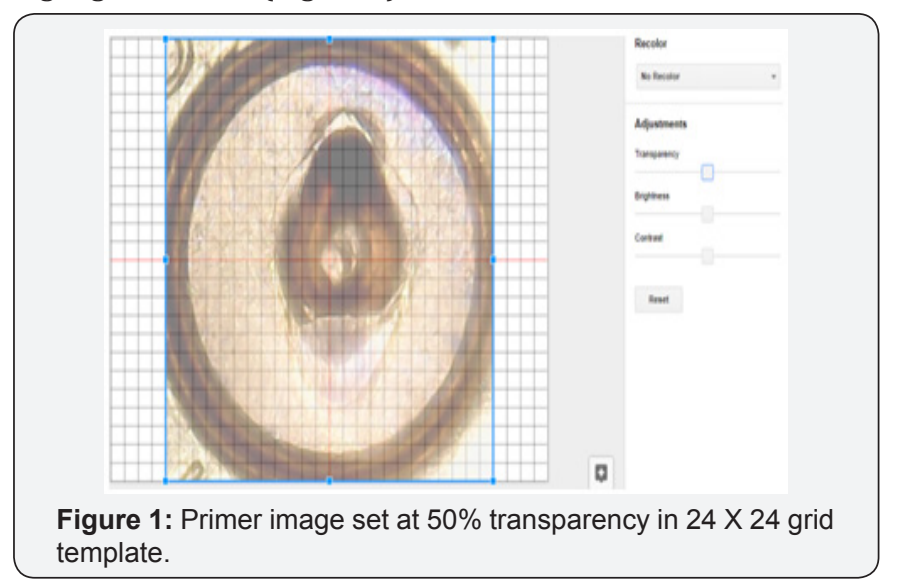

On this particular cartridge case, the Smith \& Wesson M\&P9 Shield's firing pin struck at (1.0) square or grid units. One Slide presentation was made for each individual firearm. Each of the ten tests fired primers represented one slide in each Slide presentation. The coordinates of each primer were entered into a Google Sheets spreadsheet (Google's equivalent to Excel) corresponding to each individual firearm in order for the distances to be computed based on the scale of 24 square units corresponding to $4.45 \mathrm{~mm}$. The resulting plots of firing pin strikes were then added to each firearm's slide presentation. The coordinates from firearms of the same class (make/model) were than pooled into another spreadsheet corresponding to the class.

\section{The Classification of Digital Images}

The logical progression which followed was to create various Google Drive folders corresponding to the various makes or manufacturer. While within each manufacturer folder contains the model folders, within each model folder contains the individual firearm folders labeled by serial number. Finally, a Decision Making folder for hypothetical unknown cartridge cases was created based on the previous folders of ground truth known's (Figure 2). For example, within the Decision Making folder is the first folder "FP drag present?" which then contains two folders: "Present" and "Not present." For a firearm which does not have a tilting barrel, there would not typically be any drag marks present. Most firearms without a tilting barrel are blowback action operated, as opposed to recoil action. The only instance for a blowback operated firearm to leave a drag mark would be if the firing pin served a dual purpose as an ejector (such as on the Hi-Point C9 where a lateral drag mark may be occasionally observed).

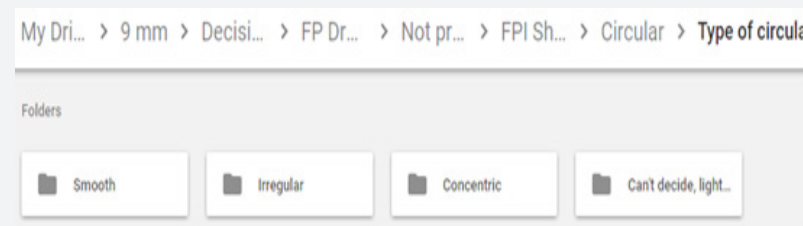

Figure 2: Google Drive filing system of cartridge case images by class characteristic features.

Following this proposed hypothetical decision tree, within "“Not present" is a folder entitled "FPI shape?" wherein lies only one folder currently: "Circular," as opposed to the more elongated "Elliptical" Glock-type firing pin impressions. Within this folder lies "Type of circular FPI?" wherein there lies four folders corresponding to "Smooth," "Irregular," "Concentric," or "Can't decide, light strike" (Figure 2). Within "Smooth" contains a folder "Size of FPA?" wherein contains two folders: "Large" or "Not large." And within "Large" is the folder corresponding to the Beretta 92FS with the characteristic large firing pin aperture mark of approximately 17 grid units $(3.15 \mathrm{~mm}$ ) which has been described previously as "primer blowback" or "pillow-like (Figure 3)." The images and the resulting variability present can then be used for a one to one comparison with the questioned cartridge cases to guide the decision maker in an objective manner akin to the sketches within the rolodex cards of Kennington's matrix system. With respect to ejector marks, rather than giving a subjective name or classification such as "oriental fan shaped," a one to one comparison can be performed (Figure 3).

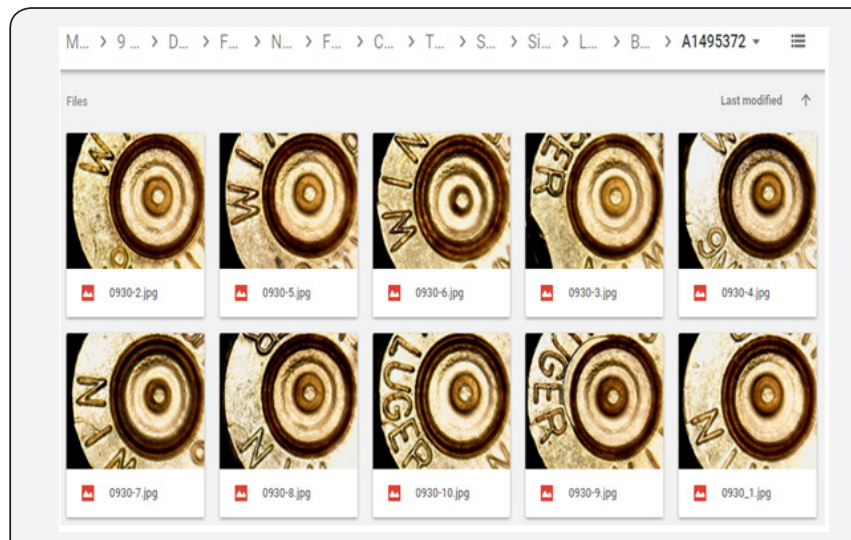

Figure 3: Decision making filing system leading to a Beretta 92FS folder, individual firearm serial number A1495372.

\section{Discussion}

There are many features which can be considered for the classification of class characteristics present on the surface of a primer. Although the optimal sequence and discriminating power of features is an object of future study, in no particular order are:
a) Presence or Absence of Firing Pin Drag
b) Shape of the Firing Pin Impression
c) Type of Breech face Impressions 

d) Type of Firing Pin Impression
e) Type of Firing Pin Aperture
f) Size of the Firing Pin Aperture
g) Firing Pin Impression's Distance off-center
h) Prominence of Firing Pin Aperture Shear Marks

A example summary of obvious feature differences between the different firearms studied can be seen in Table 1.

Table 1: Example Summary of Characteristic Features Observed.

\begin{tabular}{|c|c|c|c|c|}
\hline Feature & Firing Pin Impression & Firing Pin Aperture & Breech-face Marks & Ejector Mark \\
\hline Beretta 92 & $\begin{array}{c}\text { Smooth circular, never } \\
\text { drags }\end{array}$ & Large, ring-shaped & Non-apparent & $\begin{array}{c}\text { Large, oriental fan-shaped (if fully } \\
\text { present), at rim, incomplete if } \\
\text { intersects head stamp letters }\end{array}$ \\
\hline CZ 75 & $\begin{array}{c}\text { Concentric, circular, } \\
\text { inconsistent drag }\end{array}$ & Circular & Vertical linear & $\begin{array}{c}\text { Figure eight-shaped, at rim, can be } \\
\text { non-apparent }\end{array}$ \\
\hline HK USP & $\begin{array}{c}\text { Irregular circular, } \\
\text { inconsistent drag }\end{array}$ & Circular & Vertical linear & $\begin{array}{c}\text { Shark tooth-shaped rim, not always } \\
\text { present } \\
\text { not always present }\end{array}$ \\
\hline Sig Sauer P226 & $\begin{array}{c}\text { Circular, varying degrees } \\
\text { of drag }\end{array}$ & Circular \\
\hline S\&W M\&P9 & $\begin{array}{c}\text { Small circular, consistent } \\
\text { drag }\end{array}$ & Light bulb & Vertical linear & $\begin{array}{c}\text { Point-of-toe-shaped, at point } \\
\text { between rim and primer }\end{array}$ \\
\hline
\end{tabular}

\section{Conclusion}

When firing pin drag marks are fully present, the Sig Sauer P226 may potentially be mistaken for the Springfield XD9, if no ejector marks can be compared, however its firing pin did strike further from the center (Figure 4). Future statistical analyses are being developed to attempt to classify cartridge cases by treating the firing pin strike $x-y$ coordinates both first and foremost as a uni-variate normally distributed distance from the center of the primer independent of cartridge case orientation/rotation as well as bi-variate normally distributed $\mathrm{x}$ and $\mathrm{y}$ coordinates where the subjective state of striking to the "right and low," for example, should be supported and quantified by $x-y$ coordinates where $X_{-}$firing_pin $>0$ and Y_firing_pin $<0$ and classifying based upon the resulting means and variance-covariance of each firearm class.

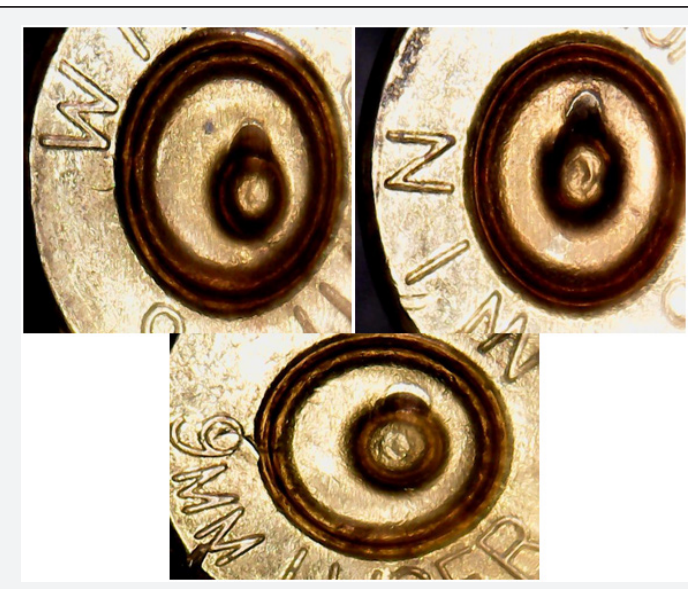

Figure 4: Both the left two cartridge cases were discharged in a Sig P226 (UU776297) while the right cartridge case was discharged in a Springfield XD9 (US141177). Only the bottom cartridge case image displays the "Sig shark tooth" ejector mark distinctly different from the XD9's ejector mark.

Besides the need for further statistical analyses, it is interesting to note Springfield's newer XD-S 9 mm has an entirely different firing pin aperture than the older XD9. The XD-S has an aperture mark similar to the signature rectangular aperture of the Glock, but more rounded and with less apparent primer shear marks, as noted by Warren and Pitts as another direction for future study. When firing pin drag marks are not present, the CZ 75 and Sig Sauer P226 can potentially be mistaken for the popular blowback operated Hi-Point C9 (Figure 4). With respect to the Smith \& Wesson M\&P9, a direction of further study with respect to the potentially mistaken for Glock 43 as identified by Warren \& Sheets (2017), it was observed to produce a round "point of toe" shaped ejector mark roughly equidistant between the primer and the rim, although this mark was sometimes obscured by the head stamp.

A potential difference may be found with respect to the position of the Glock 43 ejector mark distribution as being more towards the rim of the cartridge case than the Smith \& Wesson M\&P9. Additionally, the Smith \& Wesson M\&P9 has a smaller firing pin aperture mark of approximately 11 square units $(2.0$ $\mathrm{mm}$ ) at the widest point as opposed to the Glock 43's 13 square units $(2.4 \mathrm{~mm})$. The similarity between the Ruger SR9 to the Smith \& Wesson M\&P9 was also noted with the key difference being the SR9 having a common circular aperture mark instead of the "light bulb" shaped aperture of the M\&P9. One final observation is the larger firing pin aperture mark of the Glock 43 is reminiscent of the Kel-Tec PF9 in size (also approximately 13 square units), however the 43 had a characteristic difference in shape ("light bulb" shaped aperture) from the PF9's circular shaped aperture (similar to the Beretta 92FS with "primer flow back").

\section{Acknowledgement}

The author would like to express sincere appreciation for his Master's thesis proposal committee chair Dr. Morris and committee members Mr. Carroll and Dr. Jelsema for their insightful guidance and continued mentoring as the thesis is being executed at the time of this writing. 


\section{References}

1. Nordhoff T (1990) the Lorcin 25 ACP Caliber Pistol. AFTE Journal 22(4): 399-400

2. Thompson E (1991) Parker 45 Semiautomatic Pistol. AFTE Journal 5(2): 19-24.

3. Gieszl R (1991) Bersa 380 Semiautomatic Pistols. AFTE Journal 23(3): 862-866.

4. Carr J (1992) Lorcin L25 - A Barrel With Class. AFTE Journal 24(1): 17-21.

5. Kennington R (1992) the Matrix; 9mm Parabellum: An Empirical Study of Type Determination. Robert H Kennington 9105 NW. 29th Street, Miami, Florida, USA.
6. Dutton G (2003) Book Reviews-The Matrix: 9mm Parabellum-An Empirical Study of Type Determination \& the Matrix: 380 Autos - A Technical Guide. AFTE Journal 35(4): 384-387.

7. Oberg M (2010) A Review of SCCY (SKYY) Industries Firearms. AFTE Journal 42(3): 278-280.

8. Warren EM, Pitts SL (2017) Using Class Characteristics to Distinguish Between Cartridge Cases Fired by Glock, Smith \& Wesson Sigma, and Springfield XD Pistols. AFTE Journal 49(2): 70-78.

9. Warren EM, Sheets H (2017) Statistical Approaches to Type Determination of the Ejector Marks on Cartridge Cases. Journal of Forensic Sciences.

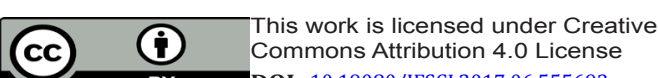

(C) DOI : $10.19080 /$ JFSCI.2017.06.555693
Your next submission with Juniper Publishers will reach you the below assets

- Quality Editorial service

- Swift Peer Review

- Reprints availability

- E-prints Service

- Manuscript Podcast for convenient understanding

- Global attainment for your research

- Manuscript accessibility in different formats

( Pdf, E-pub, Full Text, Audio)

- Unceasing customer service

Track the below URL for one-step submission https://juniperpublishers.com/online-submission.php 